\title{
An Extensive Evaluation of the Internet's Open Proxies
}

\author{
Akshaya Mani* \\ Georgetown University \\ am3227@georgetown.edu \\ David Dworken \\ Northeastern University \\ david@daviddworken.com
}

\author{
Tavish Vaidya* \\ Georgetown University \\ tavish@cs.georgetown.edu \\ Micah Sherr \\ Georgetown University \\ msherr@georgetown.edu
}

\begin{abstract}
Open proxies forward traffic on behalf of any Internet user. Listed on open proxy aggregator sites, they are often used to bypass geographic region restrictions or circumvent censorship. Open proxies sometimes also provide a weak form of anonymity by concealing the requestor's IP address.

To better understand their behavior and performance, we conducted a comprehensive study of open proxies, encompassing more than 107,000 listed open proxies and $13 \mathrm{M}$ proxy requests over a 50 day period. While previous studies have focused on malicious open proxies' manipulation of HTML content to insert/modify ads, we provide a more broad study that examines the availability, success rates, diversity, and also (mis)behavior of proxies.

Our results show that listed open proxies suffer poor availabilitymore than $92 \%$ of open proxies that appear on aggregator sites are unresponsive to proxy requests. Much more troubling, we find numerous examples of malicious open proxies in which HTML content is manipulated to mine cryptocurrency (that is, cryptojacking). We additionally detect TLS man-in-the-middle (MitM) attacks, and discover numerous instances in which binaries fetched through proxies were modified to include remote access trojans and other forms of malware. As a point of comparison, we conduct and discuss a similar measurement study of the behavior of Tor exit relays. We find no instances in which Tor relays performed TLS MitM or manipulated content, suggesting that Tor offers a far more reliable and safe form of proxied communication.
\end{abstract}

\section{KEYWORDS}

Internet Proxies, Anonymity

\section{ACM Reference Format:}

Akshaya Mani, Tavish Vaidya, David Dworken, and Micah Sherr. 2018. An Extensive Evaluation of the Internet's Open Proxies. In 2018 Annual Computer Security Applications Conference (ACSAC '18), December 3-7, 2018, San fuan, PR, USA. ACM, New York, NY, USA, 14 pages. https://doi.org/10. $1145 / 3274694.3274711$

\footnotetext{
${ }^{*}$ Equally credited authors.

Permission to make digital or hard copies of all or part of this work for personal or classroom use is granted without fee provided that copies are not made or distributed for profit or commercial advantage and that copies bear this notice and the full citation on the first page. Copyrights for components of this work owned by others than the author(s) must be honored. Abstracting with credit is permitted. To copy otherwise, or republish, to post on servers or to redistribute to lists, requires prior specific permission and/or a fee. Request permissions from permissions@acm.org.

ACSAC '18, December 3-7, 2018, San Juan, PR, USA

(C) 2018 Copyright held by the owner/author(s). Publication rights licensed to ACM. ACM ISBN 978-1-4503-6569-7/18/12 . \$15.00

https://doi.org/10.1145/3274694.3274711
}

\section{INTRODUCTION}

Open proxy servers are unrestricted proxies that allow access from any Internet user. Open proxies are fairly prevalent on the Internet, and several websites are devoted to maintaining large lists of available open proxies. In contrast to VPNs [29] and some (but not all) anonymity systems, open proxies are generally easy for users to use, requiring only minimal configuration changes (e.g., adjusting "network settings") rather than the installation of new software.

As surveyed in this paper, there is a variety of types of open proxies, with differing capabilities. This leads to variation in how proxies are used in practice. Clearly, open proxies have been (mis)used for malicious purposes, including (but not limited to) sending spam, injecting ads, and serving as stepping stones for various attacks [38, 46]. But open proxies have also been used for far less nefarious reasons, including circumventing censorship efforts. More generally, they permit accessing otherwise inaccessible information and, controversially, can be used to bypass regional content filters (e.g., to watch a sporting event that, due to licensing restrictions, would otherwise not be viewable). Importantly, along with VPNs, proxies have been suggested as a means of enhancing Internet privacy and protecting browsing history in the face of the recent expansion of data collection rights by U.S. Internet service providers [30].

An interesting related question, and one that unfortunately has not undergone rigorous study, is why the owners of open proxy servers run such services. In some instances, the proxies are due to misconfiguration or even compromise. There is a rich history of accidental open proxies, particularly for open proxies that operate as SMTP relays [43]. Still, many proxies are operated by choice. Although the rationales here are not well-understood, we posit that some operators may run proxies as a political statement about privacy and the freedom to access information.

A more menacing motivation for running a proxy is that it provides its operator with an expanded view of network traffic. Proxy operators are well situated to eavesdrop on communication, perform man-in-the-middle (MitM) attacks, and monitize their service by injecting spurious ads [46]. We note that while it is relatively difficult to operate a backbone Internet router and monitor traffic, it is not difficult to act as an open proxy and increase one's view of others' communication.

We are of course not the first to suggest that open proxies should be treated with a healthy supply of skepticism, due in large part to the ease in which proxies can be instantiated and configured to eavesdrop and manipulate communication. Most noteworthy is the recent study of open proxies by Tsirantonakis et al. [46]. There, the authors explore the extent to which open proxies modify proxied 
HTML pages to inject ads, collect user information, and redirect users to malware-containing pages. Similar to Tsirantonakis et al.'s study, we also consider the modification of retrieved webpages. This paper compliments their work by conducting a large and more broad study of the Internet's open proxies.

We perform an extensive study of the open proxy ecosystem, consisting of more than 107,000 publicly listed open proxies. We find that the vast majority $(92 \%)$ of open proxies that are publicly listed on aggregator websites are either unavailable or otherwise do not allow proxy traffic. Surprisingly, the open proxies that do allow proxy traffic have very little geographic or network diversity: five countries account for nearly $60 \%$ of the Internet's working open proxies, and $41 \%$ of such proxies are hosted by just ten autonomous systems (ASes). We also evaluate the performance of the proxies, and find a wide distribution of effective goodputs.

To understand the security implications of relaying traffic through open proxies, we present a series of experiments designed to understand a broad range of proxy behaviors. We compare the results of traffic received via direct communication with traffic that traverses through proxies. While our results indicate that the majority of open proxies seemingly operate correctly (that is, they forward the traffic unimpeded), we find a surprisingly large number of instances of misbehavior. In particular, as we discuss in more detail in what follows, we discover many instances in which proxies manipulate HTML content, not only to insert ads, but also to mine cryptocurrency (i.e., cryptojacking). Our study also discloses numerous attacks in which proxies return trojan Windows executables and remote access trojans (RATs), and conduct TLS MitM attacks.

As a point of comparison, we compare the level of manipulation observed using open proxies to that when content is fetched over Tor [23]. Over the course of our nearly monthlong experiment in which we fetched files from every available Tor exit relay, we found no instances in which the requested content was manipulated. Our results suggest that Tor offers a more reliable and trustworthy form of proxied communication.

In summary, this paper describes a large-scale and broad study of the Internet's open proxies that (i) examines the open proxy ecosystem, (ii) measures open proxy performance, (iii) measures the prevelance of previously undisclosed attacks, and (iv) relates its findings to a similar study (which we conduct) of Tor exit relay behavior. Our results demonstrate that misbehavior abounds on the Internet's open proxies and that the use of such proxies carries substantial risk.

\section{BACKGROUND}

The Socket Secure (SOCKS) protocol [34] was introduced in 1992 as a means to ease the configuration of network firewalls [33] In SOCKS, a server listens for incoming TCP connections from a client. Once connected, a client can then tunnel TCP and/or UDP traffic through the SOCKS proxy to its chosen destination(s). Since SOCKS forwards arbitrary TCP and UDP traffic, clients can use end-to-end (e2e) security protocols (in particular, TLS/SSL) through SOCKS proxies. Currently, SOCKSv4 and SOCKSv5 servers are both deployed on the Internet, with the latter adding authentication features that enforce access control. In this paper, we do not distinguish between SOCKSv4 and SOCKSv5, and use the general term SOCKS proxies to describe proxies running either version.
More commonly (see §5.1), open proxies use HTTP as a transport mechanism. This has the benefit of supporting clients whose local firewall policies prohibit non-HTTP traffic (e.g., as is sometimes the case on corporate networks). ${ }^{1}$

We distinguish between two types of proxies that rely on HTTP. The first, which we refer to simply as HTTP proxies, allows clients to specify a fully-qualified URL-with any domain name-as part of a HTTP GET request to the proxy. The proxy parses the GET URL and if the requested URL is on a different host, the proxy makes its own request to fetch the URL and forwards the response back to the client. Conceptually, a HTTP proxy is a web server that serves pages that are hosted elsewhere.

A significant disadvantage of HTTP proxies is that it is incompatible with e2e security protocols such as TLS, since it is the HTTP proxy (not the user's browser) that initiates the connection to the proxied URL. The inability to support fetching HTTPS URLs increasingly limits the use of HTTP proxies due to the growing adoption rate of HTTPS [27] on the web.

In contrast, CONNECT proxies use the HTTP CONNECT method [32] to establish e2e tunnels between the client (i.e., the browser) and the destination webserver. CONNECT proxies allow the client to specify a host and port. The proxy then initiates a TCP connection to the specified target and then transparently forwards all future TCP data from the client to the destination, and vice versa. Importantly, CONNECT proxies forward traffic at the TCP level (layer 4) and are not limited to any particular application-layer protocol. CONNECT proxies support TLS/HTTPS connections since the browser can effectively communicate unencumbered to the destination webserver and perform an ordinary TLS handshake. Example protocol interactions for HTTP and CONNECT proxies are provided in Figures 14 and 15 in Appendix A.

The network addresses (IPs and ports) of open proxies are listed on proxy aggregator sites. These sites also sometimes use the term transparent, meaning that the proxy supports at least e2e TCP tunneling; SOCKS and CONNECT proxies meet this criterion. As another dimension, aggregator sites also sometimes categorize proxies as anonymous proxies. These proxies purportedly do not reveal the client's IP address to the destination. We explore the degree to which open proxies provide anonymity in $\S 5.1$.

\section{RELATED WORK}

We are not the first to investigate the use of proxies on the Internet. Weaver et al. [52] performed a measurement study to detect the presence of transparent HTTP proxies on connections from clients to servers. They found that $14 \%$ of tested connections were proxied somewhere along the network path between the client and destination. Our work focuses on the intentional use of freely available proxies and gathers evidence of misbehavior by such proxies.

Scott et al. [40] provide insights about the use, distribution, and traffic characteristics of open proxies on the Internet. However, they do not focus on detecting any malicious behavior by open proxies, which is a core focus of this work.

Other work has investigated the misuse of proxy servers. Of note, Jessen et al. [41] deploy low-interaction honeypots to explore

\footnotetext{
${ }^{1}$ Although, as we report in $§ 5.1$, most proxies that use HTTP as a transport mechanism do not listen on ports 80 or 443 , potentially nullifying this advantage.
} 
the (ab)use of open proxies to send spam. Researchers have also examined the abuse of the Codeen CDN network [50] by looking at the requests that were forwarded by the network and mitigation strategies employed to minimize the forwarding of malicious requests $[38,51]$. While we also investigate misbehavior, we focus on uncovering malicious behavior by open proxies rather than malicious use of proxies by users.

Tyson et al. [47] study HTTP header manipulation on the Internet by various open proxies and discuss various observed factors affecting HTTP header manipulation. Huang et al. [31] show that ASes sometimes use middleboxes that interfere with HTTP traffic and inject HTTP headers across a wide range of networks including mobile and data centers. Durumeric et al. [25] examine the degree of HTTPS interception by middleboxes and argue that such interception significantly reduces security. Our work has greater scope, and focuses not only on header manipulation or HTTPS interception, but also uncovers several other forms of suspicious behavior exhibited by open proxies. Waked et al. [49] show that commercially used TLS middleboxes do not perform sufficient validation checks on SSL certificates and expose their clients to trivial attacks. We also found that some open proxies exhibit similar behavior.

Previous work has also looked at ad injection in HTML content [21, 39, 44]. Similarly, we look at content manipulation by open proxies, but consider other forms of misbehavior beyond ad injection.

Most related to this work is the recent study by Tsirantonakis et al. [46] that also looks at content manipulation by examining roughly $66 \mathrm{~K}$ open HTTP proxies on the Internet. Their work provides an in-depth analysis of different types of malicious behavior exhibited by proxies by injecting Javascript code. Their analysis shows that proxies inject Javascript aimed at tracking users, stealing user information, fingerprinting browsers, and replacing ads. Complementary to their work, we provide an in-breadth analysis of $107 \mathrm{~K}$ unique open proxies by evaluating their availability, performance, and behavior across a large spectrum of potential attacks. We look at manipulation of content by open proxies, not only through the injection of Javascript, but also by modification of different types of requested content (binary files, etc.) and through TLS MitM. Additionally, unlike existing work, we evaluate the behavior of open proxies based on different client locations. Laudably, Tsirantonakis et al. [46] built a service to detect malicious open proxies on a daily basis and to publicly report the proxies deemed unsafe. We compare our findings using this service. As an alternative to open proxies, we compare the performance of open proxies to Tor exit relays ( $\$ 9)$, and argue that Tor provides a safer means of proxied communication.

\section{METHODOLOGY \& EXPERIMENTAL SETUP}

Our study of the Internet's open proxy servers has two main goals: (i) to measure the proxies' availability, composition, and performance and (ii) to assess the degree to which proxies exhibit malicious behavior. We begin by describing the methodology used to perform our study.

We conducted our study over a 50 day period, beginning on 2018-04-12 and ending on 2018-05-31. Our measurement apparatus, described next, was installed on 16 locations (listed in Table 8 in Appendix B); these included 15 geographically diverse AWS

\begin{tabular}{lr}
\hline Proxy Aggregator & Proxies \\
\hline clarketm [10] & 6,343 \\
multiproxy.org (all) [5] & 1,524 \\
multiproxy.org (anon) [6] & 373 \\
NordVPN [9] & 29,194 \\
ProxyBroker [22] & 73,905 \\
workingproxies.org [15] & 1,250 \\
xroxy [16] & 345 \\
\hline Total (unique proxies) & 107,034 \\
\hline
\end{tabular}

Table 1: Sources of open proxies. A given proxy may be listed by more than one aggregator.

regions and an installation at Georgetown University. We term each instance a client location. Having multiple client locations allows us to determine whether proxies behave differently based on the network and/or geographic locations of the requesting client.

From each client location, an automated process performed the following steps once per day:

(1) Populate: We collect and combine lists of advertised proxies from a number of proxy aggregator sites. We augment this list with the results of running ProxyBroker [22], an open source tool for finding open proxies. In all cases, proxies were listed by aggregator sites as tuples containing an $\mathrm{IPv} 4$ address and a TCP port number. The complete list of sources of proxies is listed in Table 1 . We emphasize that the list of proxies is (re)fetched daily since, as we show in $\S 5.1$, open proxies are subject to high levels of churn.

(2) Classify: Next, from each client location, we attempt to connect to each proxy and, if successful, determine whether the proxy is a HTTP, CONNECT, or SOCKS proxy.

(3) Fetch: Finally, we request several files (URLs) from the set of proxies that we were successfully able to classify.

In more detail, during the Fetch step, we retrieve the following files from each proxy that we were able to classify, using unencrypted HTTP connections: an HTML page, a Flash object (.swf), a Windows executable (.exe), a JPEG image, a ZIP file, a Windows batch (.bat) file, a Linux/UNIX shell script (.sh), and a Java JAR archive. With the exception of the exe file (explained in more detail in §7), the URLs are hosted on web servers at Georgetown University.

For CONNECT and SOCKS proxies (i.e., the proxies that support TLS), we also request files over HTTPS from a properly configured (i.e., with a valid certificate) web server running at Georgetown University. We additionally request HTML files from https: //revoked.badssl.com/ and https://self-signed.badssl.com/ which, respectively, use revoked and self-signed certificates. The rationale for fetching content from sites with invalid certificates is discussed in §8. In all cases, we set the User-Agent HTTP request header to match that of Google Chrome version 62.0.3202.94 running on Mac OS X.

For each proxy request, we record whether the request completed. If we received a response from the proxy, we also record the HTTP status code and response string (e.g., "200 OK”) returned by the proxy, the size of the response, the content of the response, the 
MIME-type of the response (as determined by filemagic), the timeto-last-byte (TTLB) for receiving the response, the HTTP response headers, and (in the case of HTTPS requests) the certificate received.

Throughout the remainder of this paper, we use the term expected content to refer to the correct contents of the file and unexpected content to refer to content returned by a proxy that does not match the file indicated by the requested URL. A correctly functioning open proxy should thus return the expected content. As we explore in more detail below, unexpected content does not necessarily indicate malicious behavior. For instance, unexpected content could be an HTML page indicating that the proxy is misconfigured or that the proxy requires user authentication.

We remark that a weakness of our study, and one that we share with studies that examine similar (mis)behavior in anonymity and proxy services $[20,46,54]$, is that we cannot easily differentiate between manipulation that occurs at a proxy and manipulation that occurs somewhere along the path between the proxy and the destination. Our findings of misbehavior can be viewed as indications that a proxy should not be used, either because it was itself malicious or because its network location makes traffic routed through it routinely vulnerable.

\section{PROXY AVAILABILITY \& PERFORMANCE}

The number of unique proxies listed by proxy aggregator sites, over time, is shown in Figure 1. The median number of proxies listed on the aggregator sites over all days in the measurement period is 41,520 , with a range of $[38,843 ; 48,296]$. In total, during the course of our study, we indexed approximately 107,000 unique proxies that were listed on aggregator sites.

We find that more than $92 \%$ of open proxies that are advertised on proxy aggregator sites are offline or otherwise unavailable. Figure 2 plots the number of responsive proxies over time for which we were able to establish at least one connection and retrieve content. We remark that the Figure includes proxies that returned unexpected content. Across our measurement study, the median daily number of responsive proxies is 3,283 ; the medians for HTTP, CONNECT, and SOCKS proxies are 1,613; 1,525; and 74, respectively.

We use the MaxMind GeoLite2 City and ASN databases [36] to resolve each proxy's IP address to a physical location and an autonomous system (AS). Tables 2 and 4 respectively report the most frequent locations and ASes from among the proxies that are listed, responsive (i.e., respond to proxy requests), and successfully deliver the expected content at least once. There is surprisingly little geographic and network diversity among the proxies. Ten countries are responsible for nearly three-quarters of the world's working proxies, while Brazil alone is home to nearly $20 \%$ of the proxies that forward expected content.

Similarly, a handful of ASes are privy to traffic from a disproportionate amount of the open proxies. In particular, U.S.-based DigitalOcean and the Chinese No. 31 Jin-rong Street AS each host approximately $7 \%$ of the working proxies. In general, the distribution of open proxies on the Internet is very skewed, with roughly $40 \%$ of proxies confined to only 10 ASes. Moreover, open proxies are found on only a small fraction of the Internet: although more than 31,000 proxies accepted proxy requests during the course of our experiment, they resided on just 2,971 (5.8\%) of the Internet's approximately 51,500 autonomous systems [3].

We identified Squid, an open-source cacheing proxy, as the most frequent proxy software among the relays that (i) responded to our client requests and (ii) inserted a self-identifying Via or X-Via HTTP header. As shown in Table 3, Squid was used by over $85 \%$ of such proxies. Overall, we identified 130 different self-reported proxy software systems, although we note that not all proxies include Via or X-Via HTTP headers (84.43\% do not) and that such headers are easily forged and may not actually reflect the actual software.

As reported in Figure 4, approximately $62 \%$ of working proxies listened on TCP ports 8080,3128 , or 20183 . Port 8080 is an alternative port frequently used for web traffic or cacheing servers, and 3128 is the default port used by Squid. The popularity of port 20183 is surprising; it is not listed as a standard port by the Internet Assigned Numbers Authority (IANA). The standard web port, port 80 , is used by $7 \%$ of open proxies. The frequent use of ports 8080 and 3128 by open proxies provides a potential means of discovering additional potential proxies: Censys, which is based on ZMap [26] Internet scans, reports 7.3M Internet hosts listening on port 8080; Shodan lists $1.1 \mathrm{M}$ hosts with services listening on port 3128 [11]. As we discuss in $\S 10$, we limit our study to only the proxies whose addresses are listed publicly by aggregator sites, and thus for ethical reasons do not probe these additional 8.4M hosts to discover unlisted proxies.

\subsection{Performance}

We evaluate the performance of proxies by considering goodput, computed as the size of a fetched file (we use a $1 \mathrm{MiB}$ file) divided by the time taken to download it through a proxy. We consider only instances in which the response reflects the expected content (i.e., the $1 \mathrm{MiB}$ file). Figure 5 shows the range of proxies' daily average goodput for requests that yielded the expected content. Overall, the average goodput for such proxies is $128.5 \mathrm{KiBps}$, with an interquartile range (IQR) of $[39.5 ; 160.9]$ KiBps.

We also consider proxies' utility over time. A proxy that offers high goodput but functions only sporadically is not particularly useful. We define the success rate to be the fraction of proxy requests that were successfully completed and yielded the expected content. Figure 6 plots proxies' success rates as a function of their average goodput. We note that, generally, the proxies that offer the highest goodput (highlighted in the Figure with the oval) also tend to offer the highest success rates. The existence of stratified "lines" when the success rate is $1 / 3,1 / 2$, and $2 / 3$ is somewhat surprising: this indicates a regular periodicity or schedule during which these proxies are available.

\subsection{Expected vs. Unexpected Content}

Proxies do not always return the expected content. However, not all unexpected content is malicious. For example, many tested proxies return a login page or a page conveying an authentication error, regardless of the requested URL. These indicate that the listed open proxy is either misconfigured, actually a private proxy, or is some other misconfigured service. Here, we answer the question: to what extent do listed open proxies return the expected content?

For our analysis, we consider the proxies that have responded to at least one proxy request with a non-zero byte response and a 


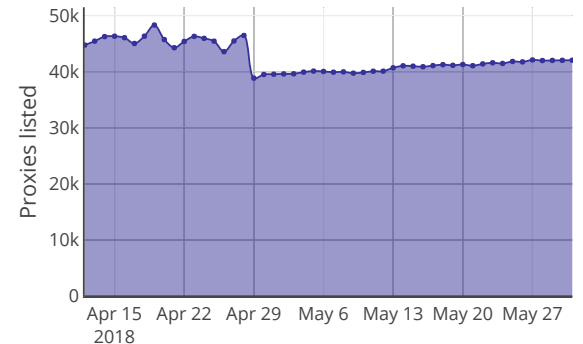

Figure 1: Number of unique open proxies listed on aggregator sites, over time.

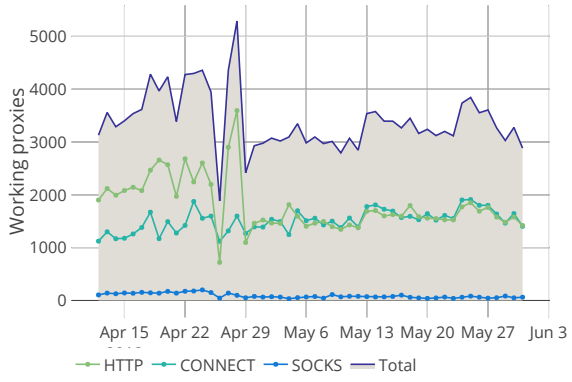

Figure 2: Working proxies, by type, over time.

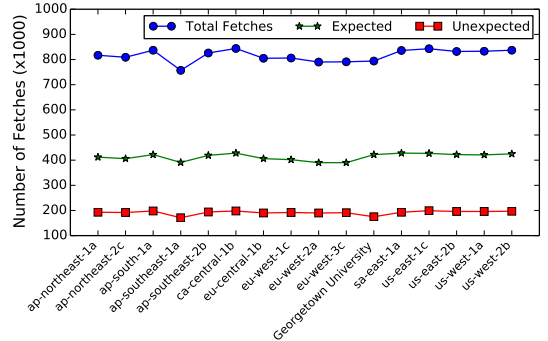

Figure 3: Total fetches and fetches with expected and unexpected content, by client location.

\begin{tabular}{lr|lr|lr}
\hline \multirow{2}{*}{ Listed } & & \multicolumn{2}{|c}{ Responsive } & \multicolumn{2}{c}{ Correct } \\
& Percent & Country & Percent & Country & Percent \\
\hline China & $18.81 \%$ & Brazil & $17.97 \%$ & Brazil & $19.48 \%$ \\
Brazil & $15.55 \%$ & China & $16.75 \%$ & China & $14.74 \%$ \\
United States & $15.19 \%$ & United States & $12.12 \%$ & United States & $12.17 \%$ \\
Indonesia & $5.95 \%$ & Indonesia & $6.77 \%$ & Indonesia & $7.15 \%$ \\
Thailand & $5.14 \%$ & Thailand & $5.73 \%$ & Thailand & $6.06 \%$ \\
Russian Federation & $4.60 \%$ & Russian Federation & $5.11 \%$ & Russian Federation & $5.40 \%$ \\
Germany & $2.53 \%$ & Singapore & $2.61 \%$ & Singapore & $2.78 \%$ \\
Singapore & $2.34 \%$ & Germany & $2.59 \%$ & India & $2.52 \%$ \\
India & $2.21 \%$ & India & $2.54 \%$ & Germany & $2.44 \%$ \\
Italy & $1.79 \%$ & Canada & $1.93 \%$ & Canada & $1.91 \%$ \\
\hline All others (count: 145) & $25.69 \%$ & All others (count: 136) & $25.65 \%$ & All others (count: 131) & $25.17 \%$ \\
\hline
\end{tabular}

\begin{tabular}{lr}
\hline Software & Percent \\
\hline squid & $87.07 \%$ \\
http_scan_by & $2.89 \%$ \\
1.1 www.santillana.com.mx & $2.29 \%$ \\
1.0 PCDN & $1.78 \%$ \\
HTTP/1.1 sophos.http.proxy:3128 & $1.74 \%$ \\
swproxy & $0.57 \%$ \\
Cdn Cache Server & $0.40 \%$ \\
MusicEdgeServer & $0.34 \%$ \\
1.1 Pxanony & $0.22 \%$ \\
1.1 j5k-8 (jaguar/3.0-11) & $0.21 \%$ \\
\hline All others (count: 120$)$ & $2.50 \%$ \\
\hline
\end{tabular}

Table 2: Locations of proxies listed on aggregator sites (left), capable of accepting proxy connections (center), and forwarding correct content at least once (right).

\begin{tabular}{|c|c|c|c|c|c|c|c|c|}
\hline \multicolumn{3}{|c|}{ Listed } & \multicolumn{3}{|c|}{ Responsive } & \multicolumn{3}{|c|}{ Correct } \\
\hline ASN & Name & Percent & ASN & Name & Percent & ASN & Name & Percent \\
\hline 4134 & No.31,Jin-rong Street & $9.34 \%$ & 14061 & DigitalOcean, LLC & $7.81 \%$ & 14061 & DigitalOcean, LLC & $7.47 \%$ \\
\hline 14061 & DigitalOcean, LLC & $6.69 \%$ & 4134 & No.31,Jin-rong Street & $7.73 \%$ & 4134 & No.31,Jin-rong Street & $6.65 \%$ \\
\hline 18881 & TELEFÔNICA BRASIL S.A & $3.55 \%$ & 18881 & TELEFÔNICA BRASIL S.A & $4.10 \%$ & 18881 & TELEFÔNICA BRASIL S.A & $4.52 \%$ \\
\hline 4837 & CHINA UNICOM China169 Backbone & $2.51 \%$ & 17974 & PT Telekomunikasi Indonesia & $2.63 \%$ & 17974 & PT Telekomunikasi Indonesia & $2.77 \%$ \\
\hline 17974 & PT Telekomunikasi Indonesia & $2.26 \%$ & 4837 & CHINA UNICOM China169 Backbone & $2.53 \%$ & 4837 & CHINA UNICOM China169 Backbone & $2.40 \%$ \\
\hline 13335 & Cloudflare Inc & $1.81 \%$ & 45758 & Triple T Internet/Triple T Broadband & $1.97 \%$ & 45758 & Triple T Internet/Triple T Broadband & $2.10 \%$ \\
\hline 16276 & OVH SAS & $1.73 \%$ & 20473 & Choopa, LLC & $1.66 \%$ & 20473 & Choopa, LLC & $1.82 \%$ \\
\hline 45758 & Triple $\mathrm{T}$ Internet/Triple $\mathrm{T}$ Broadband & $1.73 \%$ & 53246 & Cyber Info Provedor de Acesso LTDA ME & $1.62 \%$ & 53246 & Cyber Info Provedor de Acesso LTDA ME & $1.79 \%$ \\
\hline 20473 & Choopa, LLC & $1.44 \%$ & 16276 & OVH SAS & $1.52 \%$ & 16276 & OVH SAS & $1.56 \%$ \\
\hline 53246 & Cyber Info Provedor de Acesso LTDA ME & $1.38 \%$ & 31034 & Aruba S.p.A. & $1.33 \%$ & 31034 & Aruba S.p.A. & $1.45 \%$ \\
\hline - & All others (count: 3419 ) & $64.27 \%$ & - & All others (count: 2961) & $61.17 \%$ & - & All others (count: 2759) & $59.06 \%$ \\
\hline
\end{tabular}

Table 4: Most popular ASNs for proxies listed on aggregator sites (left), proxies capable of accepting connections (center), and proxies that forwarded correct content at least once (right).

2xx HTTP response code that indicates success (i.e., in [200, 299]). For each such proxy, we determine its failure rate, which we define as 1 - success rate. That is, a proxy's failure rate is the fraction of returned responses that constitute unexpected content.

Figure 7 shows the cumulative distribution (y-axis) of these proxies' failure rates ( $\mathrm{x}$-axis). Of proxies that respond to proxy requests with $2 \times x$ HTTP success codes, we find that $92.0 \%$ consistently deliver the expected content. Alarmingly, approximately $8 \%$ of the proxies at least sometimes provided unexpected content, and $3.6 \%$ of the proxies consistently returned unexpected content-all with HTTP response codes that indicate success. In $\S 6$ and $\S 7$, we explore cases in which the content has been purposefully and maliciously manipulated-for example, to return a trojan .exe file or to insert spurious ads in retrieved HTML content. Further, as shown in Figure 3, the behavior of proxies-that is, whether they returned expected or unexpected content-does not significantly vary with the location of the requesting client.

\subsection{Anonymity}

Open proxies are sometimes used as a simple method of hiding a user's IP address. We find that such a strategy is mostly ineffective, with nearly two-thirds of tested proxies exposing the requestor's IP address.

In more detail, we inspect the HTTP request headers that are sent by a proxy to the destination webserver. These include the headers added by our client toolchain (specifically: User-Agent and Accept) and those inserted by the proxy. To accomplish this, we constructed and hosted a simple web application that records HTTP request headers. We then examined the request headers that were produced when we used a client at Georgetown University to access our web application through each proxy. Of the proxies 


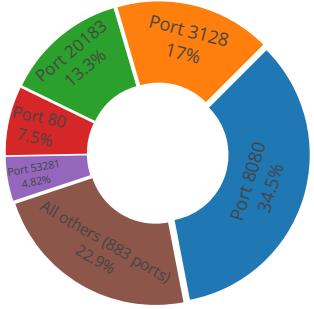

Figure 4: Proxy ports, by popularity.

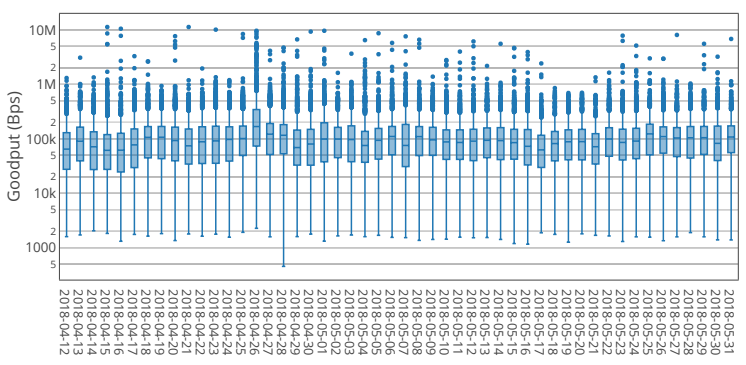

Figure 5: Proxy goodput (log-scale). Box plots depict the range of proxies' average goodput over its daily requests. The box depicts the IQR with the median; the whiskers denote $1.5 \times$ IQR. Points beyond the whiskers are outliers.

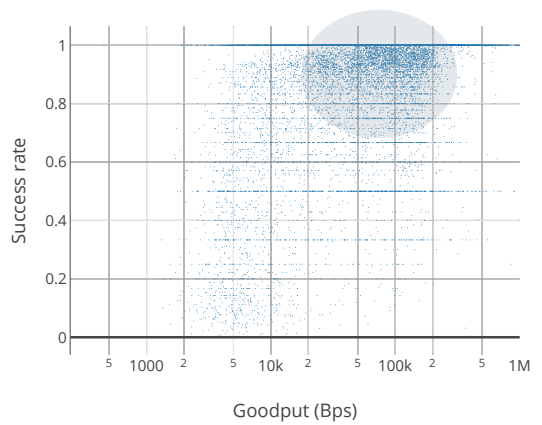

Figure 6: Proxy success rates and average goodput (log-scale). The highlighted oval denotes the densest region of the graph.

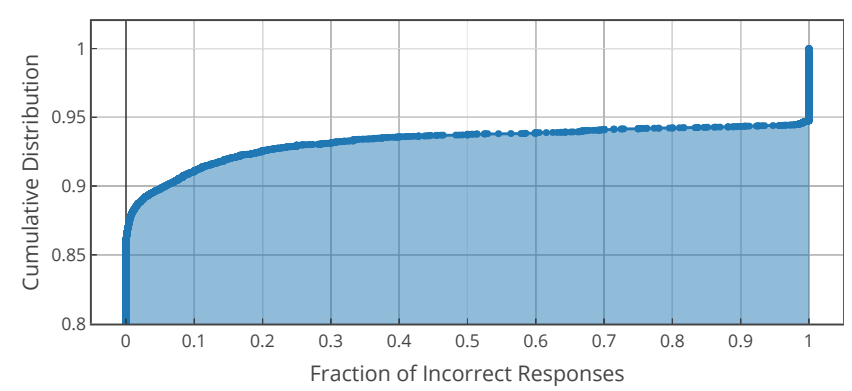

Figure 7: Cumulative distribution of the proxies' failure rate (i.e., the fraction of responses that constituted unexpected content).

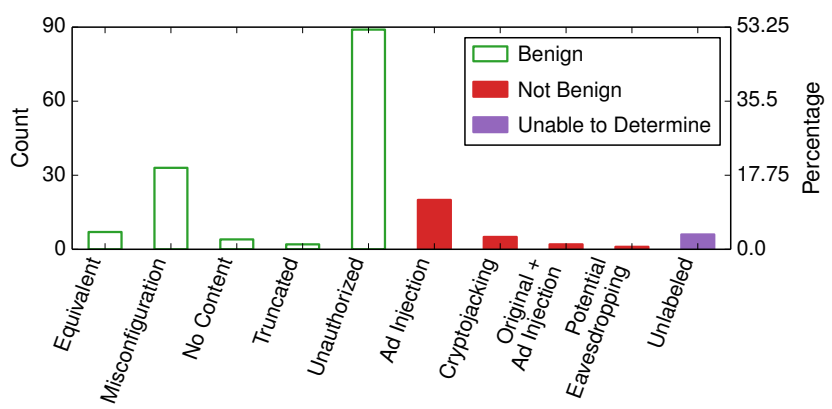

Figure 8: Classification of modified HTML retrieved on 2018-05-07.

that were able to connect to our web application $(13,740)$, we found that $66.08 \%(9,079)$ inserted at least one header (most commonly, $\mathrm{X}$-Forwarded-For) that contained the IP address of our client.

\section{HTML MANIPULATION}

We begin our study of malicious behavior among open proxies by considering the manipulation of fetched HTML content. In the absence of end-to-end SSL/TLS (i.e., the use of HTTPS), a malicious proxy can trivially either modify the web server's response or respond with arbitrary content of its choosing.

To detect such misbehavior, we fetch an HTML page via the open proxies each day between 2018-05-04 and 2018-05-31. For simplicity, we focus on the HTML pages fetched through the client at Georgetown University. We observe a median of 1,133 successful requests (i.e., completed requests with $2 x x$ HTTP response code) per day.

Overall, we find that $\mathbf{1 0 . 5 \%}$ of requests for HTML pages produced unexpected HTML content.

As noted above, not all unexpected content necessarily corresponds to malicious behavior. Since the observed fraction of unexpected HTML content is almost the same for each day of our measurement study, we perform an in-depth analysis for a random day, 2018-05-07, to determine whether the HTML manipulation could be categorized as malicious. On that date, we observe requests through 1,259 proxies, of which 169 (about 13.4\%) return unexpected HTML content. (We discard requests that did not yield 2xx success HTTP response codes.)

We manually analyze these 169 modified HTML files. Our methodology is as follows. First, we inspect each file and divide them into two categories: benign (non-malicious) files and suspicious files. The latter category represents HTML files that contain Javascript code (either directly in the file or through the inclusion of an external Javascript file).

We then collectively inspect each of these benign and suspicious files and classify them into five benign classes and four non-benign classes. This inspection process involves manually examining the files, rendering them in a browser on a virtual machine, and potentially visiting Javascript URLs that were inserted. The five classes that we posit do not indicate maliciousness are as follows:

- Equivalent: the fetched HTML renders equivalently in a web browser to the expected content. Oddly, we found instances in which HTML tag attributes were inconsequentially reordered; for instance, tags such as $<I M G \quad S R C=" A$ " ALT="B" $>$ are rewritten as $<\mathrm{IMG} \quad \mathrm{ALT}=$ "B" SRC="A" $>$.

- Misconfiguration: the retrieved content constituted error pages, often displaying "not accessible" messages.

- No Content: the page contained no content.

- Truncated: the retrieved page was a truncated version of the expected content.

- Unauthorized: the pages showed error messages such as "invalid user" or "access denied".

The identified classes of proxy misbehavior are: 


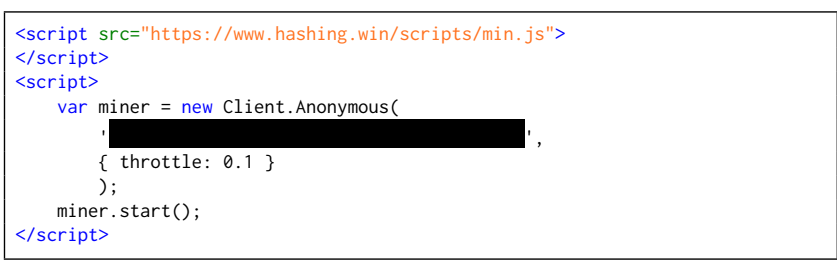

Figure 9: Injected HTML code with link to remote cryptojacking Javascript. The user identifier has been redacted.

- Ad Injection: the proxy replaced HTML content with Javascript that rendered extraneous advertisements.

- Original + Ad Injection: the returned HTML contained the expected content, but also included a single ad injection.

- Cryptojacking: the returned HTML include Javascript code that would cause the user's browser to mine cryptocurrencies on behalf of the proxy.

- Potential Eavesdropping: the retrieved page contained Javascript that caused the user's browser to visit pages from its history, potentially revealing these pages to the proxy if they were previously visited without the use of the proxy.

The classifications of the modified HTML files with their counts and percentages are shown in Figure 8 . We were unable to classify six HTML responses, described in the Figure as "Unlabeled".

Overall, we find that approximately $80 \%$ of the unexpected HTML responses are benign. Approximately $72 \%$ of the pages contained errors, likely due to private access proxies or due to misconfigured proxy software.

We find that $16.6 \%$ of unexpected HTML responses (and $2.2 \%$ of overall proxy requests for HTML content) correspond to malicious activity, including ad injection, cryptojacking, and eavesdropping. Among the malicious activity, most prominent (13\%) is ad injection.

Perhaps most interestingly, we find that about $3 \%$ of the files include Javascript that performs cryptojacking-that is, the unauthorized use of the proxy user's processor to mine cryptocurrencies in the background. Upon further inspection, we determined that each such instance uses the same injected Javascript code, shown in Figure 9.

The referenced min. js script is obfuscated Javascript. After decoding it, we determined that it is similar to Coinhive's [4] Monero ${ }^{2}$ cryptocurrency [8] mining Javascript. The 64 character long argument to the Client constructor serves as the identifier for the user to be paid in the original Coinhive setting. (We redact this argument in Figure 9 because it potentially identifies a criminal actor.) Finally, we note that all Coinhive endpoints described in the copied Coinhive script are replaced with other domains, indicating that whoever is running the infrastructure to collect the mining results is not using Coinhive's service.

\section{FILE MANIPULATION}

Over the duration of our 50 day experiment, we made more than $4.8 \mathrm{M}$ successful requests through 21,385 proxies to fetch a variety of non-HTML files (specifically, Windows .exe, Java .jar, Flash, .zip,

\footnotetext{
${ }^{2}$ Monero is advertised as a "secure, private, and untraceable" cryptocurrency [8].
}
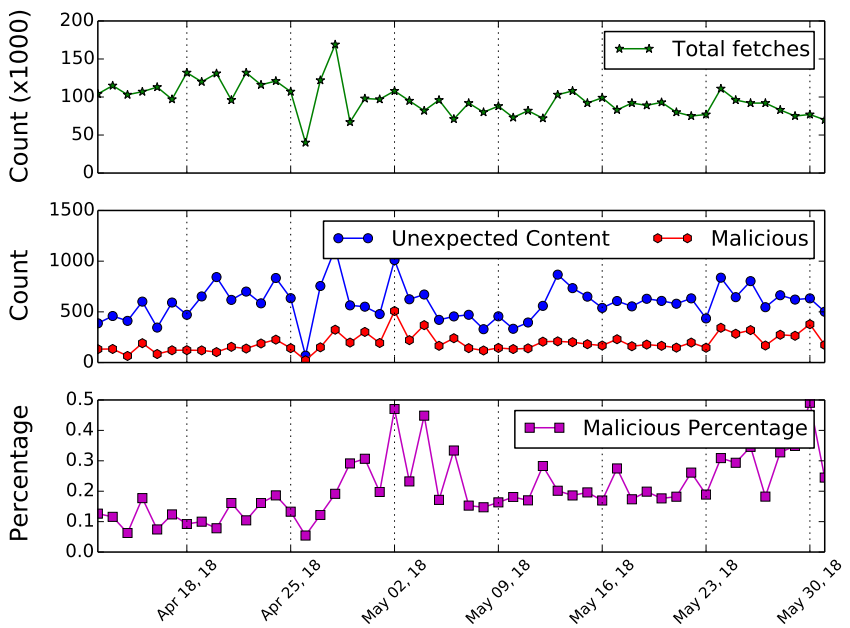

Figure 10: Per day total requests (top), unexpected responses and malicious responses (middle), and percentage of malicious responses relative to total responses (bottom).

and Windows .bat files). As before, we define a successful request to be one in which the proxy returns a $2 x x$ HTTP response code and a non-zero content size. Furthermore, we exclude HTML, plaintext, and PHP responses (as determined by filemagic) from our analysis to avoid uninteresting instances of error pages or unauthorized access pages (see §6).

Figure 10 (top) shows the frequency of total successful requests made per day, averaging approximately $97 \mathrm{~K}$ requests per day throughout the measurement period. Overall, 29,484 (0.61\%) of such requests (made by $6.76 \%$ of the proxies), constitute unexpected content.

To check whether unexpected content is malicious, we submitted all 29,484 unexpected responses to VirusTotal [14] for scanning. VirusTotal scans uploaded files using multiple antivirus tools and returns detailed analysis results, including the uploaded files determined file types. Table 5 provides a summary of our findings. Each entry for a given request and response type (determined by VirusTotal) indicates the fraction of responses with unexpected content that were flagged as malicious by VirusTotal. Note that VirusTotal (correctly) determined various responses to be HTML that were wrongly classified as non-HTML by filemagic in our initial pass of filtering out HTML pages.

To establish a baseline, we verified that all of the expected responses are not classified by VirusTotal as being malicious. That is, no single antivirus tool used by VirusTool marks the expected content as being malicious, as expected.

On the contrary, VirusTotal flags $32.84 \%(9,682 / 29,484)$ of the unexpected responses as malicious; that is, at least one of the antivirus systems used by VirusTotal flags the content as malicious. Figure 10 (middle) shows the number of unexpected and malicious responses per day. The percentage of malicious responses (relative to total success successful fetches per day) remained fairly consistent throughout our study (Figure 10 (bottom)). Across our experiment period, we find that, on average, $0.2 \%$ of daily proxy responses are classified as malicious by at least one antivirus system used by VirusTotal. 


\begin{tabular}{l|lllllllll}
\hline $\begin{array}{l}\text { Requested } \\
\text { File Type }\end{array}$ & 2 & \multicolumn{8}{c}{ Return File Type (VirusTotal) } \\
& EXE & Flash & JAR & ZIP & HTML & ISO & GZIP & XML & Unknown \\
\hline EXE & $6614 / 6713$ & - & - & - & $410 / 423$ & - & $0 / 39$ & $0 / 34$ & $12 / 234$ \\
Flash & - & $0 / 1546$ & - & - & $352 / 362$ & - & $0 / 3$ & $0 / 34$ & $0 / 32$ \\
JAR & - & - & $0 / 1075$ & $2 / 400$ & $338 / 351$ & - & $0 / 2$ & $0 / 34$ & $0 / 55$ \\
ZIP & - & - & - & $12 / 4162$ & $380 / 395$ & - & $0 / 18$ & $0 / 34$ & $0 / 176$ \\
TEXT & - & - & - & - & $436 / 446$ & $545 / 545$ & $0 / 2$ & $0 / 34$ & $23 / 10910$ \\
BAT & - & - & - & - & $556 / 574$ & - & $0 / 98$ & $0 / 34$ & $2 / 719$ \\
\hline
\end{tabular}

Table 5: Requested file types and various response file types determined by VirusTotal for unexpected responses. Each entry for a given request and response file type shows the fraction of malicious responses.

\begin{tabular}{l|cccccccc}
\hline $\begin{array}{l}\text { No. of antivirus } \\
\text { systems flagging } \\
\text { as malicious }\end{array}$ & $\mathbf{0}$ & $\mathbf{1}$ & $\mathbf{2}$ & $\mathbf{3}$ & $\mathbf{4}$ & $\mathbf{5}$ & $\mathbf{6}$ & $\mathbf{1 8}$ \\
\hline No. of Files & $\begin{array}{l}19,802 \\
(67.16 \%)\end{array}$ & $\begin{array}{l}587 \\
(1.99 \%)\end{array}$ & $\begin{array}{l}1,274 \\
(4.32 \%)\end{array}$ & $\begin{array}{l}1,508 \\
(5.11 \%)\end{array}$ & $\begin{array}{l}4,232 \\
(14.35 \%)\end{array}$ & $\begin{array}{l}1,513 \\
(5.13 \%)\end{array}$ & $\begin{array}{l}23 \\
(0.08 \%)\end{array}$ & $(1.85 \%)$ \\
\hline
\end{tabular}

Table 6: The number of files and percentage (relative to all unexpected content files) (bottom row) flagged as malicious by varying number of antivirus systems (top row) used by VirusTotal.

Table 6 shows the number of unexpected responses that are flagged as malicious, broken down by the number of antivirus tools used by VirusTotal that classified it as malicious. At the extreme, for example, we find 545 retrieved files that are considered malicious by 18 different antivirus systems.

\subsection{Detailed Findings}

In what follows, we discuss in more detail our findings of malicious proxy activity, organized by file type.

Windows Executables (.exe). Almost all (98.5\%) of unexpected responses for .exe files are classified as malicious. We find that $1.93 \%(413 / 21,385)$ of the proxies maliciously modified the .exe file at least once during our measurement period. The infections include malware from the Expiro malware family that can be used to steal personal information and provide remote access to the attacker [1], and flavors of malware from the Crypt and Artemis trojan families $[2,13]$. Table 9 in Appendix C lists the top 10 reported infections for exe files.

Flash and .jar Files. VirusTotal did not flag any of the modified Flash or Java .jar files as malicious. We are unsure of whether this indicates (i) benign (but unexplained) instances in which proxies rewrite these files, or (ii) a limitation of the scanners used by VirusTotal.

ZIP Files. For ZIP file responses with unexpected content, only a single antivirus system (McAfee-GW-Edition, v2017.2786) flagged $0.28 \%(14 / 4562)$ of them as malicious. VirusTotal did not provide any details about the infection.

HTML Files. We received HTML responses from proxies (as determined by VirusTotal) irrespective of the requested content type. Roughly $0.2 \%(44 / 21,385)$ of the proxies returned unexpected content at least once, with 43 of them returning malicious content as well. Almost $97 \%(2,472 / 2,551)$ of these HTML responses were labeled as malicious. Table 10 in Appendix C shows the reported infections and the number of HTML responses with malicious

\begin{tabular}{ccc}
\hline No. Malicious Proxies & AS Number & AS Name \\
\hline 72 & 4134 & No.31,Jin-rong Street \\
45 & 14061 & DigitalOcean, LLC \\
25 & 4837 & CHINA UNICOM China169 Backbone \\
14 & 17974 & PT Telekomunikasi Indonesia \\
9 & 56046 & China Mobile communications corporation \\
\hline
\end{tabular}

Table 7: The five ASes with the most number of malicious proxies.

code. Upon further manual examination, we found that all of the 2,472 malicious responses detected by VirusTotal contain the same Monero cryptocurrency [8] mining Javascript shown in Figure 9.

ISO Files. Surprisingly, on 545 occasions, we received ISO files when requesting a $1 \mathrm{MiB}$ text file. All of the $545 \mathrm{ISO}$ responses were exactly $1 \mathrm{MiB}$ in size, but were flagged as malicious by VirusTotal. Table 11 in Appendix $C$ shows the various infections reported by VirusTotal. We note that all 545 files were infected with the Vittalia Trojan, a rootkit for Windows. The content of 520 of the 545 ISO images was identical while the remaining 25 responses were identical to one another. Although the ISO files were clearly malicious, only $0.04 \%(9 / 21,385)$ of the proxies returned a malicious ISO response at least once.

Shell Scripts. We fetched shell scripts to determine if malicious proxies would modify (or replace) the scripts in transit. We find that $22.75 \%(211,288 / 928,431)$ of the requests for shell scripts result in responses with unexpected content. To hone-in on malicious activity, we discarded responses whose MIME-type was not "text/xshellscript"; we found 1,020 instances in which the responses were unexpected but were determined to be shell scripts. Oddly, all 1,020 instances of unexpected content correspond to just four unique responses, summarized in Table 12 in Appendix C. All of these modifications appear to be non-malicious (strangely, one frequent modification inserts the text "Pop HerePop HerePop HerePop HerePop HerePop HerePop HerePop Here" and little else) and are most likely due to misconfiguration. Overall, we did not find any evidence of malicious shell script manipulation during our measurements.

\subsection{Network Diversity and Consistency of Malicious Proxies}

For the responses that we deemed malicious, we also looked at the distribution of responsible proxies. Table 7 shows the top five ASes with the most number of proxies that performed malicious manipulations. 


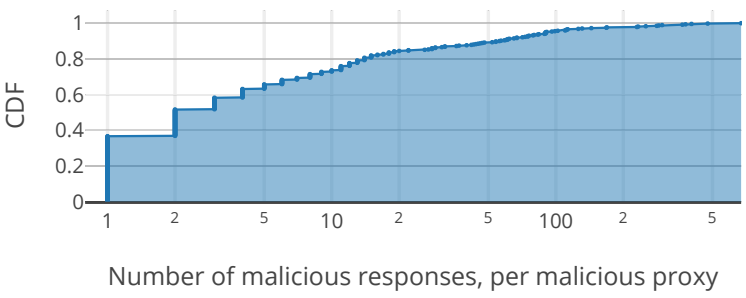

Figure 11: Consistency of malicious proxy behavior.

We also looked at the daily behavior of the top three malicious proxies over the duration of our measurement, as determined by the number of malicious responses returned. The most malicious proxy returned malicious content $100 \%$ of the time. The second and third most malicious proxies returned malicious responses $97.7 \%$ and $87.9 \%$ of the time, respectively, when they were reachable. This trend is apparent in Figure 11, which plots (in log-scale) the cumulative distribution of the number of times that malicious proxies (defined as a proxy that ever behaves maliciously) exhibit misbehavior. Here, we see that half of proxies that return malicious content do so at least twice. More than $25 \%$ of malicious proxies return at least 10 files with malicious content, while the top $10 \%$ of malicious proxies return 56 or more malicious files.

Surprisingly, none of the 469 discovered proxies that return malicious content (§7.1) are listed on the service run by Tsirantonakis et al. [46] that reports misbehaving proxies. This suggests that correctly identifying misbehaving proxies is very challenging, since proxy misbehavior may be transient and may take different forms.

\section{SSL/TLS ANALYSIS}

We find that $70 \%(14,607 / 20,893)$ of the proxies that fetch expected content at least once allow TLS traffic to pass through them. We emphasize that supporting HTTPS incurs no overhead on behalf of the proxy, since the proxy is not involved in the (end-to-end) cryptography and is merely transporting the ciphertext. It is worth noting that as of early June 2018 , more than $70 \%$ of loaded web pages were retrieved using HTTPS [35]. The lack of universal HTTPS support among the open proxies effectively forces some users to downgrade their security, bucking the trend of moving towards a more secure web.

We consider misbehavior among two dimensions: attempts at decreasing the security of the communication through TLS MitM or downgrade attacks, and alteration of the content.

SSL/TLS Stripping. We first examine whether any of the proxies rewrite HTML $<$ A $>$ link tags to downgrade the transport from HTTPS to HTTP. For example, a malicious proxy could attempt to increase its ability to eavesdrop and modify unencrypted communication by replacing all links to https://example.com with http: //example.com on all webpages retrieved over HTTP. To perform this test, we fetched via each proxy a HTML page hosted on a web server at Georgetown University over HTTP. This HTML page contained six HTTPS links. We did not find evidence of proxies stripping SSL by replacing the included links.

SSL Certificate Manipulation. In this experiment, we search for instances of SSL/TLS certificate manipulation. While interfering
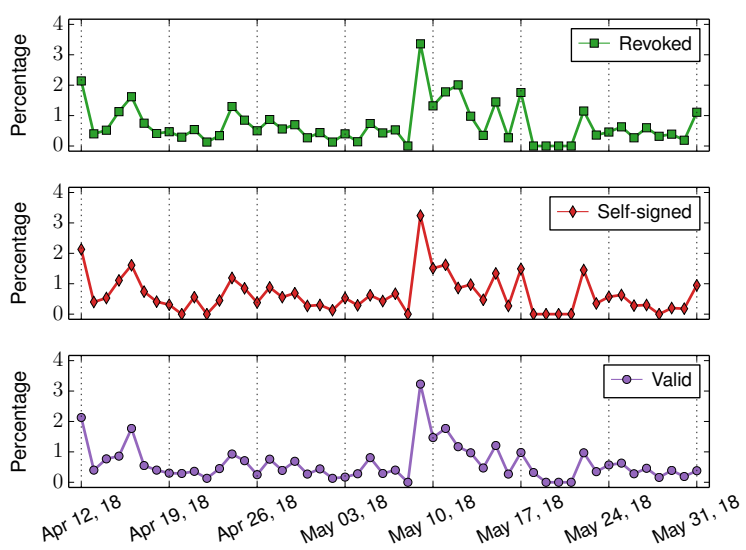

Figure 12: Percentage of proxies that return expected content but perform TLS/SSL MitM, for websites that use revoked (top), selfsigned (middle), and valid and unexpired certificates (bottom).

with an HTTPS connection would cause browser warning messages, numerous studies have shown that even security-conscious users regularly ignore such warnings $[17,42]$.

We test the proxies against two categories of domains: one with a valid and verifiable SSL certificate hosted on a web server at Georgetown University; and domains with incorrect or invalid SSL certificates (https://revoked.badssl.com, https://self-signed.badssl.com/). We include the latter category since we posit that a smart attacker might perform SSL MitM only in instances in which the connection would otherwise use revoked or self-signed certificates; in such cases, the browser would issue a warning even in the absence of the proxy's manipulation.

To detect SSL/TLS certificate manipulation, we fetch the three domains (listed above) via the open proxies each day between 2018-04-12 and 2018-05-31. For simplicity, we focus on the pages fetched through the client at Georgetown University.

Overall, we find that $1.06 \%(102 / 9,625)$ of proxies that support HTTPS perform TLS/SSL MitM by inserting a modified certificate. To determine whether any of the proxies performing SSL/TLS MitM might belong to a known botnet, we searched the SSL Fingerprint Blacklist and Dyre SSL Fingerprint Blacklist [12] for the modified certificates' fingerprints. We did not find any blacklisted certificates.

We next consider proxies that fetch the expected content but modify the SSL/TLS certificate. Such behavior suggests that the proxy is eavesdropping on HTTPS connections. The percentage of such eavesdropping proxies, per day, for the different categories of certificates is plotted in Figure 12. Overall, $0.85 \%(82 / 9,607)$ of the proxies that return expected responses appear to be eavesdropping. We did not find any evidence of proxies selectively targeting incorrect or invalid SSL certificates.

Finally, we analyze the modified TLS certificates inserted by the eavesdropping proxies when the requested site is https://revoked.badssl.com or https://self-signed.badssl.com/ (i.e., when the genuine certificate is revoked or self-signed, respectively). We find that there were 435 modified certificates from 21 unique issuers. The issuer common name $(\mathrm{CN})$ strongly suggests that $19 \%$ $(4 / 21)$ of the issuers were schools. We posit that the proxies that 
modified these certificates were operated by schools and were incorrectly configured to serve requests from any network location. Interestingly, all of the certificates inserted by these school proxies had the expected subject common name and were valid (but not normally verifiable, since the school is not a root CA). This leads to an interesting result: if the schools pre-installed root $\mathrm{CA}$ certificates on students' or employees' computers, then they are significantly degrading the security of their users. That is, they are masking the fact that requested webpages have expired or revoked certificates by replacing these invalid certificates with ones that would be verified. This is similar to the effects observed by Durumeric et al. [25] in their examination of enterprise middleware boxes, which also degraded security by replacing invalid certificates with valid ones signed by the enterprise's CA.

Performing TLS MitM also allows a malicious proxy to modify page content. To detect such behavior, using the same approach as described in $\S 6$, we analyzed HTML pages fetched over TLS via the open proxies. We did not find any malicious activity.

\section{COMPARISON WITH TOR}

Tor [23] provides anonymous TCP communication by routing user traffic through multiple relays (typically three) using layered encryption. The first relay in the path (or circuit) is the guard relay, and the final relay through which traffic exits is the exit relay. The original data transmitted is visible only at the exit relays. Therefore, unless e2e encryption is used, data can be eavesdropped by malicious or compromised exits. Prior research studies have found evidence of malicious behavior such as interception of credentials, etc., by a small fraction of Tor exit relays, especially when the traffic was not e2e encrypted [20,37,54]. We now compare the level of manipulation as observed using open proxies to that when content is fetched over Tor.

We modify Exitmap [54], a fast scanner that fetches files through all Tor exit relays. To maintain consistency with our earlier experiments, we fetched the same set of files (e.g., HTML, .exe, etc.) as described in $\S 6$ and $\S 7$ over HTTP, and accessed the same HTTPS URLs as described in $\S 8$. We fetched these files each day through every available Tor exit relay between 2018-05-06 and 2018-05-31, during which the median number of available exit relays was 722 .

Approximately $13.8 \%$ of connections and $1.8 \%$ of fetches timed out when using Exitmap. This is unsurprising since Exitmap does not perform the same (and necessary) bandwidth-weighted relay selection as the standard Tor client [23, 54].

Over our 26 day Tor experiment, we found no instance in which a Tor exit relay manipulated either file contents or SSL/TLS certificates. Comparing our results to $§ 6-8$, this strongly suggests that Tor is a more trustworthy network for retrieving forwarded content. However, since Tor exits may still passively eavesdrop (which we would not detect), we concur with the conventional wisdom that e2e encryption (i.e., HTTPS) is appropriate when using Tor.

We also compare the performance of Tor to that of the open proxies. We compute the open proxies throughput as described in $§ 5.1$. For Tor, we rely on data from the Tor Metrics Portal [45]; specifically, we use the median time taken per day to download a static file of size $1 \mathrm{MiB}$ and derive Tor's median throughput between 2018-05-06 and 2018-05-31. Figure 13 shows the Tor and open proxies' median

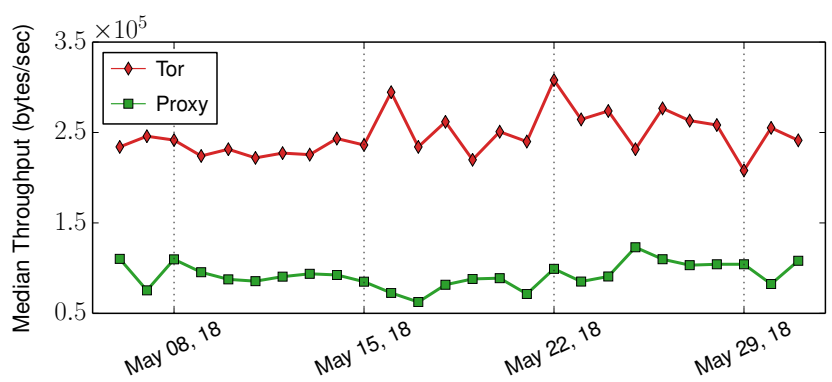

Figure 13: Tor vs. open proxy median throughput per day while fetching a static file of size $1 \mathrm{MiB}$.

throughput per day. We find that the Tor median throughput is roughly twice the open proxy throughput. This suggests that Tor performs relatively faster than the open proxies.

\section{ETHICAL CONSIDERATIONS}

We designed and conducted our measurements to minimize risk. In particular, we believe our study's design and implementation meet the criteria described in the Menlo Report [24], an ethical framework for conducting network measurements that has been widely adopted by the networking and computer security communities. The Menlo Report describes four principles of ethical research.

We achieve the principle of Respect for Persons by avoiding the collection of data belonging to individual users. In our experiments, we use open proxies largely as they are intended: we issue standard well-formed proxy requests and request only benign (nonmalicious) traffic from non-controversial websites. We record only our own traffic, and in no instance do we monitor or capture the behavior of other proxy users. We do not attempt to discover nonpublicly listed proxies by scanning the Internet. By focusing exclusively on proxies that are already publicly listed, we do not risk disclosing the existence of any previously unknown proxy.

We achieve Beneficience by minimizing potential harms while providing societal benefits (i.e., exposing the dangers of using open proxies). Unlike other studies that explicitly probe for instances of Internet censorship by requesting potentially objectionable content $[18,19,48,53]$, our measurements avoid exposing proxy operators to risk (e.g., government sanction) by retrieving only URLs that are very unlikely to be censored. Nor do our measurements consume significant resources: we request a small handful of URLs from each proxy; the average size of the content is just $177 \mathrm{KiB}$.

Our study meets the Menlo Report's fustice criterion by distributing our measurements equally across all identified open proxies.

Finally, we achieve the Respect for Law and Public Interest criterion by (i) conducting only legal queries (i.e., we are not requesting any content that is likely to be illegal or censored) and (ii) being transparent in our methods (see §4).

\section{CONCLUSION}

Open proxies provide a free and simple way to bypass regional content filters and achieve a limited degree of anonymity. However, the absence of any security guarantees for traffic passing through these proxies makes their use highly risky: users may unintentionally expose their traffic to malicious manipulations, especially when no end-to-end security mechanisms (e.g., TLS) are present. 
Our study of the Internet's open proxies-the largest conducted to date-discloses and quantifies new forms of misbehavior, reinforcing the notion that open proxies should be used with extreme caution. We found numerous instances of misbehavior, including the insertion of spurious ads and cryptocurrency-mining Javascript, TLS MitM, and the injection of RATs and other forms of malware. Moreover, we found that $92 \%$ of advertised proxies listed on open proxy aggregator sites are nonfunctional. In contrast, our nearly monthlong study of the Tor network found zero instances of misbehavior and far greater stability and goodput, indicating that Tor offers a safer and more reliable form of proxied communication.

While we remain wary about the use of open proxies, some of the risks we identify can be at least partially mitigated. Tools such as HTTPS Everywhere [7] can help reduce the risk of traffic manipulation by forcing end-to-end protections. The continued rollout of certificate transparency and similar measures will also likely reduce (but not eliminate) risk, as they thwart the certificate manipulation attacks described above.

We emphasize, however, that where e2e integrity and authenticity guarantees are not possible (e.g., for unencrypted web traffic), the use of open proxies still carries substantial risk. Given users' difficulty in adhering to safe browsing habits even in the absence of proxies [17, 28, 42], we are hesitant to recommend relying on browser-based protections to defend against malicious proxy behavior. Our findings suggest that the risks of using open proxies are plentiful, and likely far outweigh their benefits.

\section{ACKNOWLEDGMENTS}

We thank the anonymous reviewers for their valuable comments. This work has been partially supported by the National Science Foundation under grant numbers CNS-1149832 and CNS-1527401. The views expressed in this work are strictly those of the authors and do not necessarily reflect the views of the NSF.

\section{REFERENCES}

[1] Win32/Expiro, 2011. Available at https://www.microsoft.com/en-us/wdsi/threats/ malware-encyclopedia-description?Name=Win $32 \% 2$ FExpiro.

[2] Win32/Expiro, 2011. Available at https://www.securitystronghold.com/gates/ win32.trojan.crypt.html.

[3] Center for Applied Internet Data Analysis (CAIDA) AS Classification Dataset, 2018. Available at http://www.caida.org/data/request_user_info_forms/as_ classification.xml.

[4] Coinhive: A Crypto Miner for your Website, 2018. Available at https://coinhive. $\mathrm{com} /$.

[5] Free Anonymous Proxy List - All Proxies, 2018. Available at http://multiproxy org/txt_all/proxy.txt.

[6] Free Anonymous Proxy List - Anonymous Proxies, 2018. Available at http: //multiproxy.org/txt_anon/proxy.txt.

[7] HTTPS Everywhere, 2018. https://www.eff.org/https-everywhere.

[8] MONERO: Private Digital Currency, 2018. Available at https://getmonero.org/.

[9] NordVPN, 2018. Available at https://nordvpn.com/.

[10] Proxy List, 2018. Available at https://raw.githubusercontent.com/clarketm/ proxy-list/master/proxy-list.txt.

[11] Shodan, 2018. Available at https://www.shodan.io/.

[12] SSL Blacklist (SSLBL) - A list of "bad" SSL certificates, 2018. https://sslbl.abuse.ch/.

[13] Virus Profile: Artemis!10A4D2BC47D8, 2018. Available at https://home.mcafee. com/virusinfo/virusprofile.aspx?key=259866\#none.

[14] VirusTotal - Free Online Virus, Malware and URL Scanner, 2018. https://www virustotal.com/.

[15] WorkingProxies.org. The Best Free Proxy Server List on the Web, 2018. Available at http://www.workingproxies.org/plain-text.

[16] XROXY: More than Just Proxy, 2018. Available at http://www.xroxy.com/ proxylist.htm.
[17] Devdatta Akhawe and Adrienne Porter Felt. Alice in Warningland: A Large-Scale Field Study of Browser Security Warning Effectiveness. In USENIX Security Symposium (USENIX), 2013.

[18] Sam Burnett and Nick Feamster. Encore: Lightweight Measurement of Web Censorship with Cross-origin Requests. ACM SIGCOMM Computer Communication Review, 45(4):653-667, 2015

[19] John W Byers. Encore: Lightweight Measurement of Web Censorship with CrossOrigin Requests-Public Review, 2015. Available at http://conferences.sigcomm. org/sigcomm/2015/pdf/reviews/226pr.pdf.

[20] Sambuddho Chakravarty, Georgios Portokalidis, Michalis Polychronakis, and Angelos D Keromytis. Detecting Traffic Snooping in Tor using Decoys. In Recent Advances in Intrusion Detection (RAID), 2011.

[21] Taejoong Chung, David Choffnes, and Alan Mislove. Tunneling for Transparency: A Large-scale Analysis of End-to-end Violations in the Internet. In Internet Measurement Conference (IMC), 2016.

[22] Constverum. ProxyBroker, 2018. Available at https://github.com/constverum/ ProxyBroker.

[23] Roger Dingledine, Nick Mathewson, and Paul Syverson. Tor: The SecondGeneration Onion Router. In USENIX Security Symposium (USENIX), August 2004.

[24] David Dittrich, Erin Kenneally, et al. The Menlo Report: Ethical Principles Guiding Information and Communication Technology Research. Technical report, US Department of Homeland Security, August 2012.

[25] Zakir Durumeric, Zane Ma, Drew Springall, Richard Barnes, Nick Sullivan, Elie Bursztein, Michael Bailey, J Alex Halderman, and Vern Paxson. The Security Impact of HTTPS Interception. In Network and Distributed Systems Symposium (NDSS), 2017.

[26] Zakir Durumeric, Eric Wustrow, and J Alex Halderman. ZMap: Fast Internet-wide Scanning and its Security Applications. In USENIX Security Symposium (USENIX), 2013.

[27] Adrienne Porter Felt, Richard Barnes, April King, Chris Palmer, Chris Bentzel, and Parisa Tabriz. Measuring HTTPS Adoption on the Web. In USENIX Security Symposium (USENIX), August 2017.

[28] Adrienne Porter Felt, Robert W Reeder, Alex Ainslie, Helen Harris, Max Walker, Christopher Thompson, Mustafa Embre Acer, Elisabeth Morant, and Sunny Consolvo. Rethinking Connection Security Indicators. In Symposium On Usable Privacy and Security (SOUPS), 2016.

[29] Niels Ferguson and Bruce Schneier. A Cryptographic Evaluation of IPsec. Counterpane Internet Security, Inc, 3031:14, 2000.

[30] Jeff Flake. S.J.Res.34 - A Joint Resolution Providing for Congressional Disapproval under Chapter 8 of Title 5, United States Code, Of the Rule Submitted by the Federal Communications Commission Relating to "Protecting the Privacy of Customers of Broadband and Other Telecommunications Services", 2017. Public Law No: 115-22.

[31] Shan Huang, Félix Cuadrado, and Steve Uhlig. Middleboxes in the internet: a http perspective. In Network Traffic Measurement and Analysis Conference (TMA), 2017, pages 1-9. IEEE, 2017.

[32] R. Khare and S. Lawrence. Upgrading to TLS Within HTTP/1.1. Request for Comments (RFC) 2817, Internet Engineering Task Force, May 2000.

[33] D. Koblas and M. R. Koblas. Socks. In USENIX UNIX Security III Symposium, 1992.

[34] M. Leech, M. Ganis, Y. Lee, R. Kuris, D. Koblas, and L. Jones. SOCKS Protocol Version 5. Request for Comments (RFC) 1928, Internet Engineering Task Force, March 1996.

[35] LetsEncrypt. Percentage of Web Pages Loaded by Firefox Using HTTPS, 2018. Available at https://letsencrypt.org/stats/. Retrieved 9 June, 2018.

[36] MaxMind. GeoLite2 Free Downloadable Databases, 2018. Available at https: //dev.maxmind.com/geoip/geoip2/geolite2/.

[37] Damon McCoy, Kevin Bauer, Dirk Grunwald, Tadayoshi Kohno, and Douglas Sicker. Shining Light in Dark Places: Understanding the Tor Network. In Privacy Enhancing Technologies Symposium (PETS), 2008.

[38] Vivek S. Pai, Limin Wang, KyoungSoo Park, Ruoming Pang, and Larry Peterson. The Dark Side of the Web: An Open Proxy's View. SIGCOMM Comput. Commun. Rev., 34(1):57-62, January 2004.

[39] Charles Reis, Steven D Gribble, Tadayoshi Kohno, and Nicholas C Weaver. Detecting In-Flight Page Changes with Web Tripwires. In USENIX Symposium on Networked Systems Design and Implementation (NSDI), volume 8, pages 31-44, 2008.

[40] Will Scott, Ravi Bhoraskar, and Arvind Krishnamurthy. Understanding Open Proxies in the Wild. Chaos Communication Camp, 2015.

[41] Klaus Steding-Jessen, Nandamudi L Vijaykumar, and Antonio Montes. Using Low-Interaction Honeypots to Study the Abuse of Open Proxies to Send Spam. INFOCOMP Journal of Computer Science, 7(1):44-52, 2008.

[42] Joshua Sunshine, Serge Egelman, Hazim Almuhimedi, Neha Atri, and Lorrie Faith Cranor. Crying Wolf: An Empirical Study of SSL Warning Effectiveness. In USENIX Security Symposium (USENIX), 2009.

[43] Stewart Taggart. Spam Blockers Pass it On, 2001. Wired. Available at https: //www.wired.com/2001/07/spam-blockers-pass-it-on/amp/. 
[44] Kurt Thomas, Elie Bursztein, Chris Grier, Grant Ho, Nav Jagpal, Alexandros Kapravelos, Damon McCoy, Antonio Nappa, Vern Paxson, Paul Pearce, Niels Provos, and Moheeb Abu Rajah. Ad Injection at Scale: Assessing Deceptive Advertisement Modifications. In IEEE Symposium on Security and Privacy (Oakland), 2015.

[45] Tor Project, Inc. Tor Metrics Portal. https://metrics.torproject.org/, 2018.

[46] Giorgos Tsirantonakis, Panagiotis Ilia, Sotiris Ioannidis, Elias Athanasopoulos, and Michalis Polychronakis. A large-scale analysis of content modification by open http proxies. In Network and Distributed System Security Symposium (NDSS), 2018.

[47] Gareth Tyson, Shan Huang, Felix Cuadrado, Ignacio Castro, Vasile C. Perta Arjuna Sathiaseelan, and Steve Uhlig. Exploring HTTP Header Manipulation In-The-Wild. In International Conference on World Wide Web (WWW), 2017

[48] Benjamin VanderSloot, Allison McDonald, Will Scott, J. Alex Halderman, and Roya Ensafi. Scalable Remote Measurement of Application-Layer Censorship. In USENIX Security Symposium (USENIX), 2018

[49] Louis Waked, Mohammad Mannan, and Amr Youssef. To intercept or not to intercept: Analyzing tls interception in network appliances. In ACM Asia Conference on Computer and Communications Security (ASIACCS), 2018.

[50] Limin Wang, Vivek Pai, and Larry Peterson. The Effectiveness of Request Redirection on CDN Robustness. In USENIX Symposium on Operating System Design and Implementation (OSDI), 2002.

[51] Limin Wang, KyoungSoo Park, Ruoming Pang, Vivek S Pai, and Larry L Peterson. Reliability and Security in the CoDeeN Content Distribution Network. In USENIX Annual Technical Conference, General Track, pages 171-184, 2004.

[52] Nicholas Weaver, Christian Kreibich, Martin Dam, and Vern Paxson. Here be web proxies. In Passive and Active Measurement (PAM), 2014.

[53] Zachary Weinberg, Mahmood Sharif, Janos Szurdi, and Nicolas Christin. Topics of Controversy: An Empirical Analysis of Web Censorship Lists. Proceedings on Privacy Enhancing Technologies (PoPETS), 2017(1):42-61, 2017.

[54] Philipp Winter, Richard Köwer, Martin Mulazzani, Markus Huber, Sebastian Schrittwieser, Stefan Lindskog, and Edgar Weippl. Spoiled Onions: Exposing Malicious Tor Exit Relays. In Privacy Enhancing Technologies Symposium, 2014. 


\section{A EXAMPLES OF HTTP PROXY PROTOCOLS}

Example protocol interactions for HTTP and CONNECT proxies are shown in Figures 14 and 15, respectively.

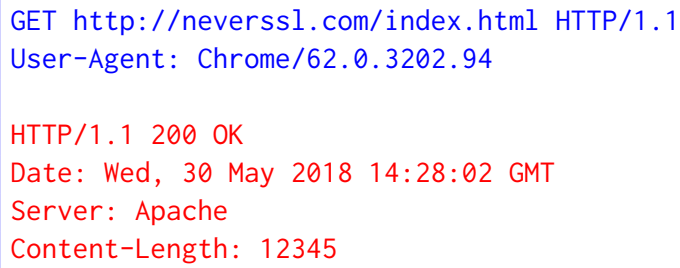

Figure 14: Example HTTP requests (in blue) and HTTP responses (in red) for HTTP proxies.

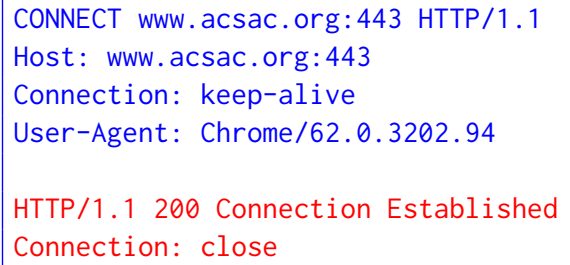

Figure 15: Example HTTP requests (in blue) and HTTP responses (in red) for CONNECT proxies.

\section{B CLIENT LOCATIONS}

\begin{tabular}{lc}
\hline Location & Downtime \\
\hline California & - \\
Canada & - \\
Frankfurt & - \\
Ireland & - \\
London & - \\
Mumbai & - \\
Paris & - \\
Ohio & - \\
Oregon & - \\
São Paulo & - \\
Seoul & - \\
Singapore & - \\
Sydney & - \\
Tokyo & - \\
Virginia & 3 days \\
Georgetown University & \\
\hline Count: 16 & \\
\hline
\end{tabular}

Table 8: Locations of clients and the number of days during the measurement period in which that client did not participate.

\section{FILE MANIPULATION INFECTIONS}

\begin{tabular}{lr}
\hline Infection & Frequency \\
\hline W32/Behav-Heuristic-CorruptFile-EP & $72.2 \%(4781 / 6614)$ \\
W32.FamVT.ExpiroPC.PE & $63.7 \%(4218 / 6614)$ \\
Heur.Corrupt.PE & $56.0 \%(3704 / 6614)$ \\
malicious_confidence_100\% (D) & $31.9 \%(2111 / 6614)$ \\
static engine - malicious & $26.8 \%(1777 / 6614)$ \\
Suspicious_GEN.F47V0511 & $23.2 \%(1535 / 6614)$ \\
BehavesLike.Win32.Generic & $18.1 \%(1200 / 6614)$ \\
malicious_confidence_90\%(D) & $9.5 \%(632 / 6614)$ \\
Trojan.Crypt9 & $7.6 \%(503 / 6614)$ \\
Artemis & $3.08 \%(204 / 6614)$ \\
\hline
\end{tabular}

Table 9: Top 10 infections reported by VirusTotal for Windows executable response file type with unexpected content.

\begin{tabular}{lr}
\hline Infection & No. of Infected Files \\
\hline HTML/ScrInject.B & 2471 \\
JS:Miner-Q & 2415 \\
Trojan.Script & 2283 \\
Trojan.ScrInject!8.A & 1158 \\
\hline
\end{tabular}

Table 10: Infections reported by Virus Total for HTML response file type with unexpected content.

\begin{tabular}{lr}
\hline Infection & No. of Infected Files \\
\hline Application.Bundler.iStartSurf & 545 \\
HEUR/AGEN.1004062 & 545 \\
Riskware.Win32.StartSurf.evlkna & 545 \\
SoftwareBundler:Win32/Prepscram & 545 \\
Trojan.Vittalia.13684 & 545 \\
W32/StartSurf.AU.gen!Eldorado & 545 \\
Win32:Evo-gen & 545 \\
heuristic & 545 \\
malware & 545 \\
not-a-virus:HEUR:AdWare.Win32.Generic & 545 \\
Application.Bundler.iStartSurf.DO & 545 \\
Application.Bundler.iStartSurf.DO (B) & 545 \\
Packed-UT!5694618272F0 & 520 \\
Packed-UT!4809C4235C2C & 25 \\
\hline
\end{tabular}

Table 11: Infections reported by VirusTotal for ISO response file type with unexpected content. 


\begin{tabular}{llr}
\hline Unique Files & Modification & Count \\
\hline 1 & OC + HTTP Headers + Truncated OC & 1 \\
2 & OC + 0 and Newline Character & 713 \\
3 & OC $+<$ body $><$ html $>$ & 8 \\
4 & OC +9 lines of harmless HTML & 298 \\
\hline Total & & 1020 \\
\hline
\end{tabular}

Table 12: Unique responses for modified shell script. $\mathrm{OC}=$ Original Content 